\title{
Study on an Improved Algorithm for Optimization of PID Parameters
}

\author{
http://dx.doi.org/10.3991/ijoe.v12i02.5050 \\ Shaofei $\mathrm{Wu}$ \\ Wuhan Institute of Technology, Wuhan, China
}

\begin{abstract}
The improved ant colony algorithm is a hybrid algorithm consisting of a genetic algorithm and ant colony algorithm convergence. Through introduction of a gauss mutation, we achieved the goal of improving an ant colony algorithm. Using a coal-fired power plant unit as the main steam temperature controlled object, we designed a PID controller based on an improved ant colony algorithm. Setting of the PID parameters by the $Z$ - $N$ method carried on a comparative analysis of the main steam temperature control system. Simulation results show that PID optimization based on the improved ant colony algorithm can greatly improve the dynamic performance of the control system. So we verified the sophistication and effectiveness of the algorithm.
\end{abstract}

Index Terms-Improved ant colony algorithm; Genetic algorithm; Gauss mutation; PID; Optimization.

\section{INTRODUCTION}

Ant colony algorithm ${ }^{[1]}$ is a kind of hot bionic algorithm following new bionic algorithms such as the simulated annealing method, genetic algorithm, tabu search algorithm, and artificial neural network. Genetic algorithm $^{[2]}$ is a kind of search algorithm based on space, through selection, heredity and mutation operation, and Darwin's theory of survival of the fittest, simulating the natural evolution to find a solution for the problem. Genetic algorithm and ant colony algorithm are both very good artificial intelligence algorithms, including the good abilities of global optimization. However, a lot of research results show that the single use of a genetic algorithm or an ant colony algorithm to solve a variety of optimization problems makes it impossible to find a satisfied solution. ${ }^{[3]}$

Based on this, we put forward a kind of improved ant colony algorithm combined with a new ant colony algorithm and genetic algorithm.

\section{The TEMPERATURE CONTROL SyStem}

For the main steam temperature of a $300 \mathrm{MW}$ power plant $^{[4]}$ of an object in a cascade control system, object properties are shown in Figure 1.

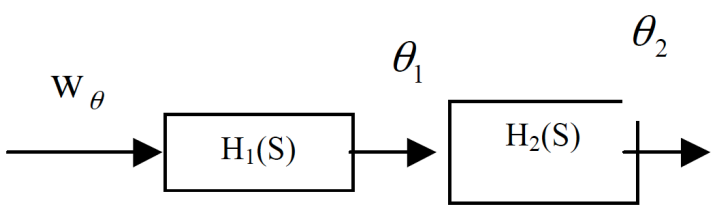

Figure 1. Object properties
The controlled object is the big inertia, a lag based on the scale of the object. In the control system, the object model is:

$$
\begin{aligned}
& H_{1}(S)=\frac{-1.20}{1+33 S} e^{-6 s} \\
& H_{2}(S)=\frac{8}{1+90 S} e^{-53 s}
\end{aligned}
$$

By the main steam temperature control system design principle, ${ }^{[5]}$ the principle diagram of the control system is shown in Figure 2.

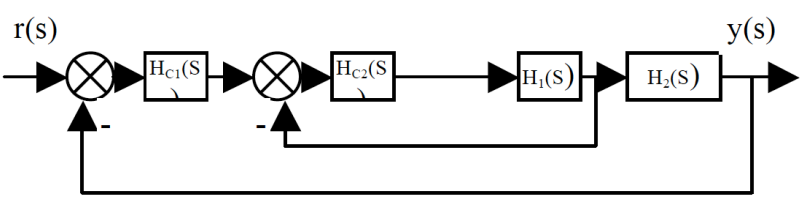

Figure 2. The main steam temperature control system controlled by cascade.

\section{PID SetTing BASED On IMPROVED Ant COLONY ALGORITHM}

\section{A. The Basic Principles of Improved Ant Colony Algorithm}

The ant colony optimization (ACO) algorithm ${ }^{[1]}$ is a population based on a heuristic bionic evolutionary algorithm; this algorithm adopts distributed parallel computing, a positive feedback mechanism, is easy to combine with other methods, has stronger robustness, and is especially suitable for solution of the combinatorial optimization problem (COP). The gauss mutation operation makes use of Gaussian distribution, which has better local search ability of the ant algorithm to solve a weak ability for local optimization. Using the decision variable set in the upper and lower bounds of the variance and mean results in meeting the conditions of Gaussian distribution. Through the operation of the Gauss mutation variance attenuation with increasing iterations, we can shrink it to the size of the global search. Simply, because the basic ant colony algorithm is easy to put into a local optimum and through the Gauss mutation operation to overcome this shortcoming, we can find the global optimal point of high efficiency and high precision. 


\section{B. PID Setting Steps Based on the Improved Algorithm}

Optimization of the PID parameters is a continuous domain of the function optimization problems in applications of ant colony algorithms. Based on the improved ant colony algorithm of nonlinear continuous function optimization, the specific steps are as follows:

1) Make the constraint function into the objective function and determine all the decision variables;

2) Estimate the scope of each decision variable;

3) Ants position initialization, pheromone initialization, set up each subspace of the decision variables within the scope of the decision variables as random to determine each ant in the various decision variable initial subspaces;

4) The number of iterations $\mathrm{nc}=\mathrm{nc}+1$;

5) Ant $\mathrm{j}=\mathrm{j}+1$;

6) The decision variables $\mathrm{i}=\mathrm{i}+1$;

7) In the case of decision variable I, choose subinterval of $j$, and randomly select candidate values within the subinterval through Gauss mutation to generate new candidate values;

8) $\mathrm{i}$ is greater than the number of decision variables, or turn 6);

9) $\mathrm{j}$ is greater than the number of ants, or turn 5);

10) Update all of the decision variables of the subspace of the pheromone; new candidates generate by comparing the objective function value and the old candidate values, use fitness good new candidate values to replace old candidate values;

11) Optimize requirements, or turn to 4 );

12) Output the result.

\section{Optimization of the PID Controller Model}

The improved ant colony algorithm and PID combination can optimize the three parameters of PID controller online $\mathrm{Kp}, \mathrm{Ti}, \mathrm{Td}$. The PID control system based on an improved ant colony algorithm structure is shown in Figure 3

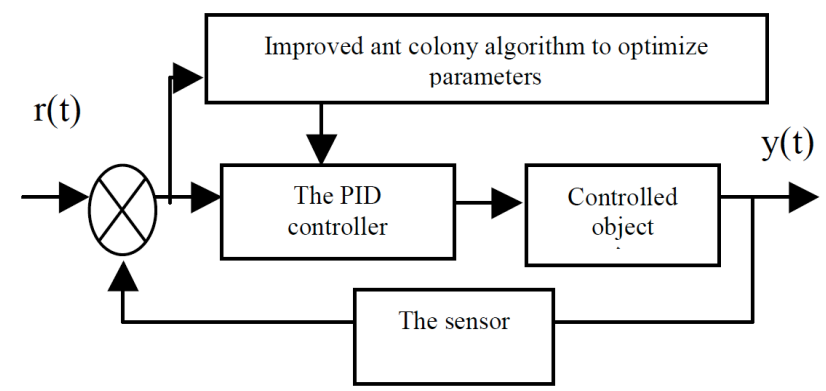

Figure 3. The principle diagram of the control program of improved ant colony algorithm

\section{The Choice of Optimal Performance Index}

Based on an integral square error (ISE) performance index ${ }^{[2]}$, namely:

$$
I S E=\int_{0}^{\infty} e^{2}(t) d t
$$

\section{The Simulation Test Study}

\section{A. The Simulation Parameters}

By using the $\mathrm{Z}$ - $\mathrm{N}$ setting of the PID parameter control method to calculate the parameters of the PID regulator $\mathrm{Kp}=0.4508, \mathrm{Ti}=170.0, \mathrm{Td}=20.0$.

\section{B. Improved Ant Colony Algorithm Parameter Settings}

The ant colony algorithms of variable initializations are the number of iterations $\mathrm{k}=20$. The number of ant colonies of ants $\mathrm{N}=100$; pheromone volatilization coefficients are $\rho=0.8$; the information enhancement coefficient $\mathrm{Q}=1$; and ants crawling speed is 0.4 . Set the upper and lower bounds of the three control parameters as:

$\mathrm{Kp} \in(0.2,10), \mathrm{Ti} \in[0.002,0.008] ; \mathrm{Td} \in[17,21]$, $\alpha=1, \beta=1.5$. After running the program in MATLAB 7.0 , the setting of the control parameters based on the improved ant colony algorithm is as follows: $\mathrm{K}_{\mathrm{p}}=0.2147$, $\mathrm{T}_{\mathrm{i}}=0.0022, \mathrm{~T}_{\mathrm{d}}=19.8433$

\section{The Results of Simulation and Analysis}

By using the $\mathrm{Z}$ - $\mathrm{N}$ setting, the system response curve is shown in Figure 4: the $\mathrm{Z}-\mathrm{N}$ method of setting the PID parameters, adjust the time $\mathrm{t} \mathrm{s}=78 \mathrm{~s}$, overshoot $\sigma \mathrm{p}=$ $21 \%$. With the improved genetic algorithm setting, the step response curve is shown in Figure 5: adjust the time $=55 \mathrm{~s}$, overshoot $\sigma \mathrm{p}=0 \%$.

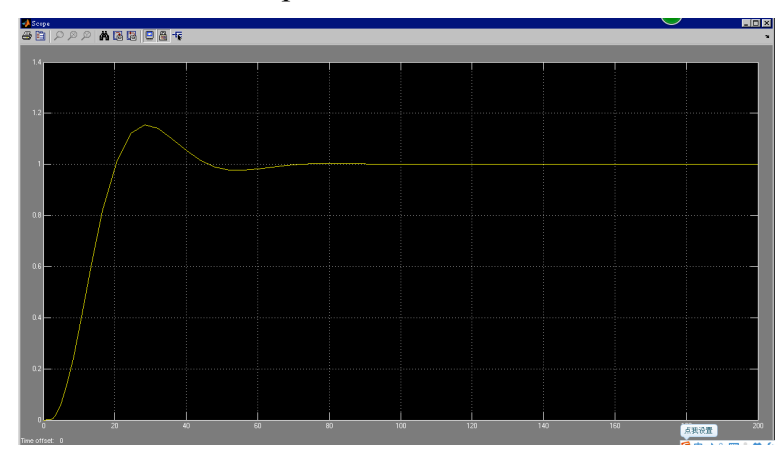

Figure 4. $\mathrm{Z}-\mathrm{N}$ setting system response curve.

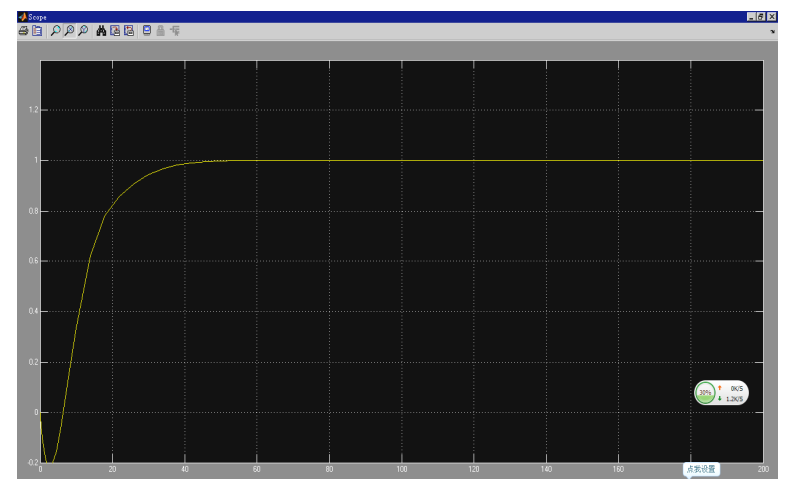

Figure 5. PID control system response curve based on optimizing of improved ant colony algorithm 
PAPER

STUDY ON AN IMPROVED ALGORITHM FOR OPTIMIZATION OF PID PARAMETERS

TABLE I.

PID PARAMETERS SETTINGS BETWEEN Z -N OPTIMAL AND VACA CONTRAST

\begin{tabular}{l|ccccc}
\hline Algorithm & $\mathbf{K}_{\mathbf{P}}$ & $\mathbf{T}_{\mathbf{i}}$ & $\mathbf{T}_{\mathbf{d}}$ & $\mathbf{t}_{\mathbf{s}}$ & $\boldsymbol{\sigma}_{\mathrm{p}} \mathbf{0}$ \\
\hline $\mathrm{Z-N}$ & 0.4508 & 170.0 & 20.0 & $78 \mathrm{~s}$ & $21 \%$ \\
\hline VACA & 0.2147 & 0.0022 & 19.8433 & $55 \mathrm{~s}$ & $0 \%$ \\
\hline
\end{tabular}

An improved ant colony algorithm for a PID parameters optimization performance index, with a $\mathrm{Z}-\mathrm{N}$ setting method of comparison of the results are shown in Table I.

\section{CONCLUSION}

Combining an ant colony algorithm and genetic algorithm, using a new method of improved ant colony algorithm, in coal-fired power plant unit for a main steam temperature controlled object, we designed a PID controller based on an improved ant colony algorithm. And setting the PID parameters by the $\mathrm{Z}-\mathrm{N}$ method has carried on the comparative analysis of the main steam temperature control system. Simulation results show that using the Z-N method of setting the main regulator control system to adjust time, the overshoot amount is larger, and based on an improved ant colony algorithm to optimize the control system has good dynamic performance.

\section{REFERENCES}

[1] Yang Yong, Song Xiaofeng Wang Jianfei. “Ant colony algorithm to solve the continuous space optimization problem." Control and decision, Vol.18, Issue.5, 2003, pp. 573-577.

[2] Zhang yi,Liang Yanchun. "Ant colony algorithm to solve the parameters optimal choice analysis." Computer application research, Vol.24, Issue. 8, 2007, pp. 70-72

\section{AUTHORS}

Shaofei Wu is with School of Computer Science and Engineering and the Hubei Province Key Laboratory of Intelligent Robot, Wuhan Institute of Technology, Wuhan, 430000, China (e-mail: wushaofei@wit.edu.cn).

The work is supported by Doctor Fund of Wuhan Institute of Technology (201210304007). Submitted 17 September 2015. Published as resubmitted by the author 23 January 2016. 